\title{
Traditional knowledge of wild edible plants with special emphasis on medicinal uses in Southern Shan State, Myanmar
}

\author{
Thant Shin ${ }^{1,2}$, Kazumi Fujikawa ${ }^{3}$, Aung Zaw Moe ${ }^{4}$ and Hiroshi Uchiyama ${ }^{1 *}$ (D)
}

\begin{abstract}
Background: Myanmar is one of the hotspots of biodiversity and is a rapidly developing country. Performing floristic research in Myanmar is an urgent issue, and ethnobotanical studies of wild edible plants (WEPs) will provide new information on natural plant resources.

Method: Ethnobotanical data were collected in three villages with different historical backgrounds in Southern Shan State, Myanmar. A total of 19 key informants were interviewed, and specimens were collected in the fields with the participation of key informants in June-July 2015. Group discussions were organized during 2016 and 2017 to reinforce the information on use of WEPs. DNA barcoding was used to facilitate species identification.

Results: A total of 83 species from 44 families of angiosperms were recorded as WEPs. Most of the species were used as wild vegetables ( 47 species), followed by fruits and nuts (31 species). Eighteen WEPs were consumed as medicinal foods. Differences in use of WEPs between the communities of the villages were observed. The age class of 30-39 years was more familiar with the environments where they could collect WEPs and had more knowledge of WEPs than did the older groups. The use of Elaeocarpus floribundus as an edible oil is a very interesting tradition.

Conclusion: WEPs play an important role in the livelihood of local communities. The indigenous society has maintained traditional knowledge of the WEPs. Historical background, land use system and surrounding vegetation could have effects on the variation in the traditional uses of WEPs. Increasing awareness of the importance of WEPs will encourage the conservation of traditional knowledge of indigenous populations.
\end{abstract}

Keywords: DNA barcode, Ethnobotany, Myanmar, Medicinal plants, Wild edible plants

\section{Background}

Wild edible plants (WEPs) are defined as plant species collected in the wild to be consumed as food or drink. Although important nutrients for humans are available from WEPs, it was argued that the use of WEPs is decreasing in urban-style cooking $[1,2]$. Being an important source of energy and micronutrients, WEPs can increase the diversification of human diets [3, 4]. WEPs were also important food sources during famine, when the normal food supply was disrupted [2]. Currently, humans focus on a limited number of plant species for staple food, neglecting the importance and usefulness of WEPs. This restriction can lead to global

\footnotetext{
* Correspondence: uchiyama.hiroshi@nihon-u.ac.jp

${ }^{1}$ College of Bioresource Sciences, Nihon University, 1866 Kameino, Fujisawa,

Kanagawa 252-0880, Japan

Full list of author information is available at the end of the article
}

food shortage and loss of the knowledge about WEPs $[5,6]$. Documentation of WEPs is important for the identification of food sources from the surrounding environment, and WEPs are serving as gene pools for genetic improvement of crops to achieve higher productivity, disease resistance and compatibility with global climate change [7]. WEPs also have the potential to be developed into new crops through domestication [8]. Moreover, the nutritional and medicinal properties of WEPs are increasingly recognized [9-12]. The traditional uses of plant resources and the lifestyles of rural communities have changed in accordance with the switch from subsistence farming/hunting and gathering to profit-oriented agricultural systems [9]. The local food tradition is a kind of cultural expression, and a loss of traditional knowledge of WEPs implies a loss of cultural identity [13]. 
Myanmar is one of the hotspots of biodiversity [14, 15] and is a rapidly developing country. Describing the flora of Myanmar is an urgent issue, and ethnobotanical studies will provide new information on natural plant resources $[16,17]$. A total of 135 ethnic groups are officially recognized in Myanmar [18]. These indigenous people regard natural resources as essential to their culture and well-being [19], but they are not truly interested in the conservation of natural resources. Furthermore, local communities are rarely allowed to participate in decision-making processes concerning the impact of ecosystem changes, and their dependence on plant resources is not adequately considered in formulating the strategies of rural development [20]. In Myanmar, quite a few ethnobotanical studies have been carried out mostly emphasizing medicinal and financial value [21-24].

Field research was carried out in three villages, each with different historical backgrounds, namely Eden, Myin Ka, and Pin-sein-pin, located in the western part of Southern Shan State (Table 1 and Fig. 1). Southern Shan State is situated on the Shan Plateau, which rises to the east from the central basin of Myanmar and occupies the eastern half of the country. The average elevation of the plateau is approximately $900 \mathrm{~m}$. The average annual precipitation is between $1900 \mathrm{~mm}$ and $2000 \mathrm{~mm}$, and the average daily temperature is $22{ }^{\circ} \mathrm{C}$ [25]. The ethnic groups residing in the study area are Danu, Taung-yoe, Shan, Bamar, Pao, Kayan and Kayin. The majority of people in the villages of Myin $\mathrm{Ka}$ and Pin-sein-pin are Danu and Taung-yoe. The major ethnic group in Eden village is Kayan. According to the folklore of the villagers, the old village of Myin Ka was established during the Bagan Era (9th-13th centuries) one mile away from the current village location. In the late nineteenth century, the old village was destroyed because of ethnic conflicts in the area and was moved to the current location. The villagers of Eden migrated from Kaya State because of civil war in their former area and established the village in 1990.

This study focused on the local communities in Southern Shan State to document their traditional knowledge of and practices involving WEPs. In the present study, a DNA barcoding technique was used to facilitate species identification. DNA barcoding is a microgenomic identification system in which a short, standard DNA region that is universally present in the target lineages is analyzed [26, 27]. With the help of DNA barcoding techniques, species identification can be facilitated in the ethnobotanical studies

Table 1 Basic information of the three villages studied

\begin{tabular}{|c|c|c|c|}
\hline & Myin Ka & Pin-sein-pin & Eden \\
\hline Establishment & $\begin{array}{l}\text { Before late nineteenth century (Moved from nearby old village, } \\
\text { one mile away). }\end{array}$ & $\begin{array}{l}\text { Early nineteenth } \\
\text { century }\end{array}$ & 1990 \\
\hline Location & N: 2034'54.3", E: 96³4'52.7" & $\begin{array}{l}\mathrm{N}: 20^{\circ} 58^{\prime} 54.3^{\prime \prime}, \mathrm{E}: 96^{\circ} \\
37^{\prime} 54.9^{\prime \prime}\end{array}$ & N: $19^{\circ} 55^{\prime} 31.9^{\prime \prime}$, E: $96^{\circ} 25^{\prime} 58.1^{\prime \prime}$ \\
\hline Above sea level & $1422 \mathrm{~m}$ & $1736 \mathrm{~m}$ & $353 \mathrm{~m}$ \\
\hline Habitat & Evergreen forest & Evergreen forest & Deciduous forest \\
\hline $\begin{array}{l}\text { Linear distance } \\
\text { to the nearest } \\
\text { town }\end{array}$ & $10 \mathrm{~km}$ to Kalaw, Shan State & $\begin{array}{l}8 \mathrm{~km} \text { to Pindaya, } \\
\text { Shan State }\end{array}$ & $40 \mathrm{~km}$ to Tatkone, Mandalay Division \\
\hline $\begin{array}{l}\text { Population/ } \\
\text { Households }\end{array}$ & $563 / 140$ & $535 / 127$ & $410 / 68$ \\
\hline Ethnic Group & Majority: Taung-yoe, Danu Minority: Bamar, Shan & $\begin{array}{l}\text { Majority: Danu } \\
\text { Minority: Bamar, Pao }\end{array}$ & Majority: Kayan Minority: Kayin, Bamar \\
\hline Religion & Buddhism & Buddhism & Christian and Buddhism \\
\hline Public Facility & One library, one primary school, one buddhist monastery & $\begin{array}{l}\text { One primary school, } \\
\text { one library, one } \\
\text { kindergarten }\end{array}$ & $\begin{array}{l}\text { One middle school, one church, one } \\
\text { monastery, one kindergarten }\end{array}$ \\
\hline Subsistence & $\begin{array}{l}\text { Rice cultivation (ca. } 60 \text { acres) Cultivation of vegetables and } \\
\text { fruits: ginger, cabbage, cauliflower, egg plants, tomato, chili, } \\
\text { chayote, carrot, orange, avocado, pear (ca } 150 \text { acres) }\end{array}$ & $\begin{array}{l}\text { Plantation of tea } \\
\text { leaves, Ju, pea, } \\
\text { cabbage, potato }\end{array}$ & $\begin{array}{l}\text { Cultivation of turmeric, chili, banana, hill } \\
\text { rice Collection of bamboo shoots and } \\
\text { other forest products }\end{array}$ \\
\hline $\begin{array}{l}\text { Home garden } \\
\text { products }\end{array}$ & Ginger, orange, avocado, pear & Tea leaves & Mango, banana, jack fruit \\
\hline Live stock & Buffalo, chicken, cattle, pig & Cattle, chicken & Pig, chicken \\
\hline Drinking water & Natural springs (connected with pipe line) & $\begin{array}{l}\text { Collection of rain } \\
\text { water with collection } \\
\text { tanks }\end{array}$ & Natural springs (connected with pipe line) \\
\hline Electricity & From national grid & No electric supply & $\begin{array}{l}\text { Small hydropower generators constructed } \\
\text { on the stream }\end{array}$ \\
\hline
\end{tabular}




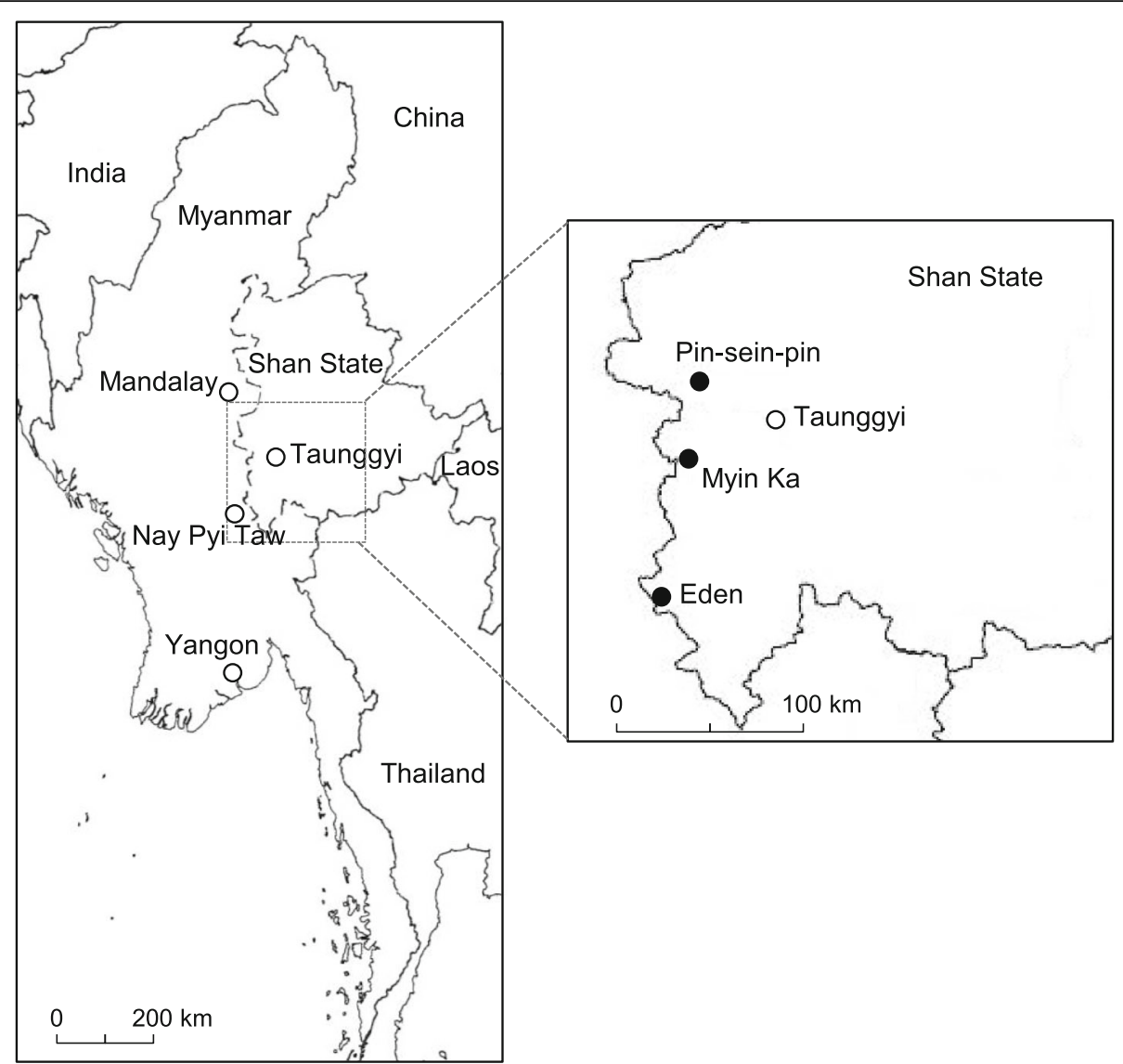

Fig. 1 Map of study area location

$[28,29]$ and floristic studies of unexploited tropical regions [30]. A total of 83 species from 44 families of angiosperms have been documented as WEPs in the study area. The indigenous societies, who have been living in the area for a long time, could have a considerable amount of traditional knowledge of WEPs.

\section{Methods}

Interviews and collection of plant specimens

First, meetings were organized with villagers, and the objectives of the research were explained. Consent was obtained before carrying out the ethnobotanical survey. The ethical guidelines of the International Society of Ethnobiology were strictly obeyed [31].

The ethnobotanical data were first collected from 19 key informants who were selected for their reputation of being specialists in the use of WEPs. The information on WEPs was gathered from interviews and fieldwork. The ages of the key informants ranged from 30 to 57 years, and one informant was female. All key informants were farmers. The field surveys were carried out in June-July 2015, and voucher specimens and the leaves for DNA analysis were collected. During the field surveys, the vernacular name, growth habitat and folk use of each species were recorded. Interviews and field sampling were carried out with one individual informant at a time to avoid the incorporation of the knowledge from other villagers. Voucher specimens were deposited in the herbaria of the Makino Botanical Garden (MBK), Japan, and the Forest Research Institute (RAF), Myanmar.

After gathering ethnobotanical data from the key informants, group discussions were organized again in 2106 and 2017. In addition to the 19 key informants, 23 other informants were invited for the group discussion. These 23 informants were selected for their willingness to participate in this study, ability to work together in the field, and reputation for being knowledgeable on the use of WEPs. Therefore, a total of 42 villagers participated in this project, including 5 female informants. A total of 13 , 12 and 17 informants from the villages of Eden, Myin Ka and Pin-sein-pin, respectively, participated in the group discussion. The age of informants ranged from 23 to 82 years, and the group included 37 farmers, one small shop owner, one forester and three herbalists. We also gathered information on WEPs with a group of informants by using the "walk-in-the-wood" method [32]. 


\section{DNA barcoding and species identification}

Total DNA was extracted from dried leaf tissue by the CTAB method. Approximately $20 \mathrm{mg}$ of dried leaf tissue was ground in a $2 \mathrm{~mL}$ microcentrifuge tube using TissueLyser (Qiagen, Hilden, Germany). The sample was incubated with $2 \times \mathrm{CTAB}$ solution at $65{ }^{\circ} \mathrm{C}$ for $30 \mathrm{~min}$. After chloroform (chloroform:isoamylalcohol $=24: 1$ ) treatment for $30 \mathrm{~min}$, the mixture was centrifuged for $10 \mathrm{~min}$ at $3000 \mathrm{rpm}$. The aqueous layer was transferred to a new $1.5 \mathrm{~mL}$ microcentrifuge tube, and then the DNA was precipitated with ethanol. The pellet was dissolved with TE buffer. PCR amplification of the $r b c L$ region followed the procedure of the CBOL Plant Working Group [33]. rbcLa_F and rbcLa_R were used as primers. For the PCR, GoTaq Green Master Mix (Promega, Madison, WI, USA) was used. Amplification was performed in a TaKaRa PCR Thermal Cycler Dice Touch (Takara, Kusatsu, Japan) programmed for one cycle of $95{ }^{\circ} \mathrm{C}$ for $5 \mathrm{~min}$; 30 cycles of $95{ }^{\circ} \mathrm{C}$ for $30 \mathrm{~s}, 55^{\circ} \mathrm{C}$ for $30 \mathrm{~s}$, and $72{ }^{\circ} \mathrm{C}$ for $1 \mathrm{~min}$; and one cycle of $72{ }^{\circ} \mathrm{C}$ for $5 \mathrm{~min}$. The PCR products were resolved by electrophoresis in $1.0 \%$ agarose gels stained with GelGreen (Wako, Osaka, Japan). DNA fragments were recovered using the Wizard SV Gel and PCR Clean-up System (Promega, Madison, WI, USA) according to the manufacturer's instructions. Cycle sequencing was performed using a BigDye Terminator v3.1 Cycle Sequencing Kit and an ABI 3130 Genetic Analyzer (Thermo Fisher Scientific, Waltham, MA, USA).

The Basic Local Alignment Search Tool (BLAST) program was used as a tool to search for similar DNA sequences from the database of the National Center for Biotechnological Information (NCBI). Among the compared sequences, the species with the most similar sequences were used to guide species identification. The final identification of specimens was determined by cross referencing with specimens housed at MBK, the type specimen images available on the JSTORE Global Plants website (https://plants.jstor.org), and the online herbarium catalog of the Royal Botanic Gardens, Kew (http://apps.kew.org/ herbcat/navigator.do). The plant descriptions in the Flora of China and Flora of Thailand were consulted. The scientific names of species were assigned in accordance with The Plant List (http://www.theplantlist.org). The uses of WEPs and medicinal plants were classified into categories according to the standards developed by the Royal Botanic Gardens, Kew [34].

\section{Results}

\section{Diversity of WEPs in the study area}

A total of 153 specimens were collected as WEPs from the three villages in this study. Of these, 138 specimens were identified to the species level and consisted of 83 species. Araceae, Fabaceae and Moraceae were the major families with the largest number of species (5 species each). Zingiberaceae, Asteraceae, Myrtaceae and Rutaceae included four species. Ethnobotanical data such as vernacular names, collection sites and folk uses are listed in Table 2. Among the 83 species, most are indigenous in Myanmar, but some of them originated outside of Asia: Annona cherimola, Casimiroa cf. edulis, Coffea arabica and Psidium guajava escaped from plantations; and Alternanthera philoxeroides, Bassia scoparia, Bidens biternata, Crassocephalum rubens, Marah macrocarpa, Oxalis latifolia, Physalis angulata, Physalis pubescens and Solanum torvum are naturalized.

In total, 34, 57 and 47 species were used as WEPs in the villages of Eden, Myin Ka and Pin-sein-pin, respectively (Fig. 2). Although most of the WEPs were used with a single vernacular name within a village, eight species had two or three local names. Physalis angulata and Leea indica were used without local names in Eden village. Two species of Amorphophallus and three species of Syzygium were used with the same local name in all three villages. Among the 39 WEPs used in more than one village, eight species were used with different local names. Phyllanthus emblica had different local names in all three villages.

\section{Use of WEPs}

Although villagers collected WEPs for their own consumption, nine species, namely, Acacia concinna, Acacia pennata subsp. kerrii, Archidendron jiringa, Cheilocostus speciosus, Lasia spinosa, Markhamia stipulata, Oroxylum indicum, Phyllanthus emblica and Telosma cordata, were collected for selling at local markets.

A total of 47 species from 25 families were used as vegetables. All five species in Araceae were used as wild vegetables. Based on the interviews with villagers, the most preferred species for use as wild vegetables were Phyllanthus emblica, Rotheca serrata and Docynia indica. P. emblica and R. serrata were also used as medicinal plants in the study area. In addition to collecting it from the wild, the villagers planted P. emblica in their home gardens for their own consumption.

A total of 31 species from 23 families were used for their fruits and nuts. Species in Myrtaceae, Moraceae and Sapindaceae were frequently used as fruit plants. The most preferred fruit plants were Docynia indica, Ficus semicordata, F. auriculata, Myrica esculenta and Archidendron jiringa. In addition to collecting it from the wild, small-scale planting of Docynia indica was observed not only for fruits but also for fuel wood. The whole plants of Oxalis latifolia were eaten by children as a snack in Myin Ka village. This species is a perennial weed originating from Central and South America [35] and is growing in home compounds of the village. From the other two villages, no species were reported as a snack for children. 
Table 2 List of identified wild edible plants used by villagers in this study

\begin{tabular}{|c|c|c|c|}
\hline Taxon & $\begin{array}{l}\text { Village }^{a} \text { and } \\
\text { vernacular name }\end{array}$ & Use & Voucher number (Collection site ${ }^{a}$ ) \\
\hline \multicolumn{4}{|l|}{ Amaranthaceae } \\
\hline $\begin{array}{l}\text { Alternanthera } \\
\text { philoxeroides (Mart.) } \\
\text { Griseb. }\end{array}$ & $\begin{array}{l}\text { M, P: Shwe-kana- } \\
\text { phot, Ka-na-phot }\end{array}$ & Food and Medicine: shoot for salad, consumed for body swollen & TS0366 (P) \\
\hline Amaranthus viridis $\mathrm{L}$. & M: Hin-nu-nwe & Food: tender leaves as vegetable & TS0020 (M) \\
\hline $\begin{array}{l}\text { Bassia scoparia (L.) } \\
\text { A.J.Scott }\end{array}$ & M: Ta-byat-se & Food: shoots and tender leaves as vegetable & TS0019 (M) \\
\hline \multicolumn{4}{|l|}{ Anacardiaceae } \\
\hline $\begin{array}{l}\text { Mangifera sylvatica } \\
\text { Roxb. }\end{array}$ & E: Taw-tha-yat & $\begin{array}{l}\text { Food and Construction: fresh fruits pounded as salad with other } \\
\text { ingredients, sliced fresh fruits for sour taste }\end{array}$ & TS0809 (E) \\
\hline \multicolumn{4}{|l|}{ Annonaceae } \\
\hline Annona cherimola Mill. & M, P: Aw-le & Food: fruits edible & TS0043 (M) \\
\hline \multicolumn{4}{|l|}{ Apiaceae } \\
\hline Centella asiatica (L.) Urb. & E, M, P: Myin-kwar & $\begin{array}{l}\text { Food and Medicine: tender leaves as salad, paste of leaves } \\
\text { prepared lotion for sore throat, cold infusion of leaves as eye } \\
\text { drop }\end{array}$ & TS0165, TS0293 (M) \\
\hline Oenanthe cf. javanica & M: Za-lae & Food: tender leaves as salad, cooked as traditional curry & TS0138, TS0166 (M) \\
\hline
\end{tabular}

(Blume) DC.

Apocynaceae

Telosma cordata (Burm. E, M, P: Gwe-tauk

Food and Medicine: tender leaves for soup with chicken,

f.) Merr.

consumed food as medicine for alcohol dependence

TS0190 (M)

Araceae

Amorphophallus cf.

Amorphophallus

Hook.f.

Arisaema erubescens

(Wall.) Schott

Colocasia esculenta (L.) Schott

E: Pain-ga-nan; M, P: $\mathrm{Pa}$

E, M: Za-yit

Lasia spinosa (L.)

Thwaites

Araliaceae

Macropanax dispermus (Blume) Kuntze

Asteraceae

Bidens biternata (Lour.) Merr. \& Sherff

Crassocephalum rubens (Juss. ex Jacq.) S.Moore

Dichrocephala integrifolia (L.f.) Kuntze Laggera alata Nanth.

Bignoniaceae

Markhamia stipulata (Wall.) Seem.

Oroxylum indicum (L.) Kurz

P: Hlan-kwa

M: Sein-zar-myat-

lone
E: Ma-Iwa$$
\text { fried as vegetable }
$$
E, M, P: Kyaung-shar Food and Medicine: flowers and fruits for vegetable dish, fruits as salad, farmented fruit, boiled and pounded fruits as salad

E, M, P: Wa-U

Food: stem and bulb as vegetable: young stem cooked as vegetable, bulb boiled and grounded to make konjac (Wa-u)

E, M, P: Wa-U

Food: stem and bulb as vegetable: young stem cooked as vegetable, bulb boiled and grounded to make konjac (Wa-u)

M, P: Wa-u-pho

Food and Medicine: stem and bulb as vegetables, bulb boiled and eaten for constipation

P: Tha-yat-kin, Ka-la- Food, Construction, Fuelwood and Medicine: shoot as vegetable
kin
dish and salad, food consumed for retention of gasses in bowel

E: Nu-su; P: Taw-bi- Food: shoot as vegetable, tender leaves for salad

Food and Medicine: shoots and tender leaves for soup, food consumed as medicine at postpartum period for mother

and Medicine: shoots fried with eggs, good for swollen used over knife injuries

petiole prepared soup with Kin-pun-chin, Sue-pote and Zayit; sap used externally for allergy caused by insects

Food: tender shoot cooked as soup, cooked with fish, boiled shoot as salad with fish paste

TS0728/1 (E)

Thant Shin (abbreviate as TS hereafter) $0618(\mathrm{P})$

TS0284 (M); TS0599 (P)

TS 0040, S0137, TS0277 (M)

TS0757 (E)

TS0590, TS0619, TS0645 (P)

TS0563 (P)

TS00684 (P); TS0776 (E)

TS0038 (M)

TS0078 (M)

TS0735 (E)

TS0105 (M); TS0743 (E) 
Table 2 List of identified wild edible plants used by villagers in this study (Continued)

\begin{tabular}{|c|c|c|c|}
\hline Taxon & $\begin{array}{l}\text { Village }^{a} \text { and } \\
\text { vernacular name }\end{array}$ & Use & Voucher number (Collection site ${ }^{a}$ ) \\
\hline & & $\begin{array}{l}\text { with other ingredients, young leaves prepared salad, and } \\
\text { consumed orally for tinnitus }\end{array}$ & \\
\hline \multicolumn{4}{|l|}{ Burseraceae } \\
\hline $\begin{array}{l}\text { Protium serratum (Wall. } \\
\text { ex Colebr.) Engl. }\end{array}$ & E: Kadi & Food and Construction: fruits eaten fresh & TS0704 (E) \\
\hline \multicolumn{4}{|l|}{ Celastraceae } \\
\hline $\begin{array}{l}\text { Celastrus paniculatus } \\
\text { Willd. }\end{array}$ & $\begin{array}{l}\text { M, P: Taung-bort-lu- } \\
\text { lin }\end{array}$ & $\begin{array}{l}\text { Food and Medicine: tender leaves prepared for soup, diet food } \\
\text { for healthy life }\end{array}$ & TS0230 (M) \\
\hline \multicolumn{4}{|l|}{ Combretaceae } \\
\hline $\begin{array}{l}\text { Terminalia bellirica } \\
\text { (Gaertn.) Roxb. }\end{array}$ & E, M, P: Thit-seint & Food: seeds eaten fresh & TS0702 (E) \\
\hline \multicolumn{4}{|l|}{ Costaceae } \\
\hline $\begin{array}{l}\text { Cheilocostus speciosus } \\
\text { (J.Koenig) C.D.Specht }\end{array}$ & $\begin{array}{l}\text { E, M: Pha-lan-taung- } \\
\text { hwa }\end{array}$ & $\begin{array}{l}\text { Food and Medicine: shoot fried with vegetable oil and other } \\
\text { ingredients, cooked for soup, cooked with bamboo shoot and } \\
\text { meat, decotion of whole plant taken orally for dysentery }\end{array}$ & TS0037 (M); TS0732 (E) \\
\hline \multicolumn{4}{|l|}{ Cucurbitaceae } \\
\hline $\begin{array}{l}\text { Marah macrocarpa } \\
\text { (Greene) Greene }\end{array}$ & M: Kin-mon-tee & Food: fruits as vegetable & TS0094 (M) \\
\hline $\begin{array}{l}\text { Momordica subangulata } \\
\text { Blume }\end{array}$ & M: Taw-hin-khar & $\begin{array}{l}\text { Food and Medicine: fruits and leaves as vegetable, consumed as } \\
\text { appetizer }\end{array}$ & TS0114, TS0276 (M) \\
\hline \multicolumn{4}{|l|}{ Ebenaceae } \\
\hline Diospyros kaki L.f. & M: Tae, Tel; P: Tel & Food: ripe fruits edible & TS0142 (M) \\
\hline \multicolumn{4}{|l|}{ Elaeagnaceae } \\
\hline $\begin{array}{l}\text { Elaeagnus griffithii } \\
\text { Servettaz }\end{array}$ & P: Mat-Iwat, Myat-lu & Food: ripe fruits edible & TS0359, TS0480 (P) \\
\hline \multicolumn{4}{|l|}{ Elaeocarpaceae } \\
\hline $\begin{array}{l}\text { Elaeocarpus floribundus } \\
\text { Blume }\end{array}$ & M: Sein-sar-blue-pan & $\begin{array}{l}\text { Food and Fuellwood: seeds used to extract edible oil, seed } \\
\text { edible }\end{array}$ & TS0183 (M) \\
\hline $\begin{array}{l}\text { Elaeocarpus stipularis } \\
\text { var. siamensis (Craib) } \\
\text { Coode. }\end{array}$ & M: Sein-se-ba-lu & Food: ripe fruits edible & TS034 (M) \\
\hline \multicolumn{4}{|l|}{ Fabaceae } \\
\hline $\begin{array}{l}\text { Acacia concinna (Willd.) } \\
\text { DC. }\end{array}$ & E, M; Kin-mon-chin & $\begin{array}{l}\text { Food and Shampoo: decoction of fruits for shampoo, tender } \\
\text { leaves prepared soup with bean, prepared salad, fried with fish } \\
\text { paste }\end{array}$ & TS0139 (M) \\
\hline $\begin{array}{l}\text { Acacia pennata subsp. } \\
\text { kerrii I.C.Nielsen }\end{array}$ & $\begin{array}{l}\text { E: Sue-pote, Sue- } \\
\text { pote-kyi; M, P: Sue- } \\
\text { pote }\end{array}$ & $\begin{array}{l}\text { Food: tender leaves cooked as soup, cooked with fish, cook with } \\
\text { meat, fried with egg, vegetable dish }\end{array}$ & TS0736 (E) \\
\hline $\begin{array}{l}\text { Archidendron jiringa } \\
\text { (Jack) I.C.Nielsen }\end{array}$ & E, M, P: Da-nyin & Food: boiled seeds & TS0710, TS0811 (E) \\
\hline Bauhinia purpurea $\mathrm{L}$. & $\begin{array}{l}\text { E: Swe-daw; P: Kha- } \\
\text { lat }\end{array}$ & Food: tender leaves as soup with potato & TS0419 (P); TS0856 (E) \\
\hline Bauhinia variegata $\mathrm{L}$. & P: Kha-la & Food: leaves as salad. Seed edible as pulses & TS0629 (P) \\
\hline \multicolumn{4}{|l|}{ Fagaceae } \\
\hline $\begin{array}{l}\text { Lithocarpus lindleyanus } \\
\text { (Wall. ex A.DC.) } \\
\text { A.Camus }\end{array}$ & M: Thit-al-sein & Food and Constructiion: roasted seed edible & TS0240 (M) \\
\hline \multicolumn{4}{|l|}{ Lamiaceae } \\
\hline $\begin{array}{l}\text { Rotheca serrata (L.) } \\
\text { Steane \& Mabb. }\end{array}$ & $\begin{array}{l}\text { M: Yin-byar; P: Hin- } \\
\text { byar, Hin-khar }\end{array}$ & $\begin{array}{l}\text { Food and Medicine: tender leaves and flowers as vegetable and } \\
\text { salad, young leaves consumed as salad for retention of gasses in } \\
\text { bowel, and diarrhea, cream of rhizome used as lotion on } \\
\text { abdomen for retention of gasses in bowel, roots fermented }\end{array}$ & $\begin{array}{l}\text { TS0005, TS0082, TS0209, TS0279 } \\
\text { (M); TS0387, TS00494, TS0591, } \\
\text { TS0606 (P) }\end{array}$ \\
\hline
\end{tabular}


Table 2 List of identified wild edible plants used by villagers in this study (Continued)

\begin{tabular}{|c|c|c|c|}
\hline Taxon & $\begin{array}{l}\text { Village }^{a} \text { and } \\
\text { vernacular name }\end{array}$ & Use & Voucher number (Collection site ${ }^{a}$ ) \\
\hline & & together with jaggery and consumed orally for loss of sleep & \\
\hline \multicolumn{4}{|l|}{ Lauraceae } \\
\hline $\begin{array}{l}\text { Cinnamomum tamala } \\
\text { (Buch.-Ham.) T.Nees \& } \\
\text { Eberm. }\end{array}$ & P: Thit-kya-poe & $\begin{array}{l}\text { Food and Medicine: leaf for spice in traditional curry, dry bark } \\
\text { powder consumed orally as blood tonic, dry leaf powder used } \\
\text { as inhalant at postpartum period for mother }\end{array}$ & TS0678 (P) \\
\hline Laurus cf. nobilis L. & M, P: Lae-lu & Food: leaves for spices & TS0483 (P) \\
\hline \multicolumn{4}{|l|}{ Lythraceae } \\
\hline $\begin{array}{l}\text { Duabanga grandiflora } \\
\text { (DC.) Walp. }\end{array}$ & E: Ga-zaw & Food and Construction: fruits edible & TS00716 (E) \\
\hline \multicolumn{4}{|l|}{ Melastomataceae } \\
\hline $\begin{array}{l}\text { Osbeckia nepalensis } \\
\text { Hook. f. }\end{array}$ & P: Shar-pyar-tee & Food: fruits edible & TS00631(P) \\
\hline \multicolumn{4}{|l|}{ Moraceae } \\
\hline Ficus auriculata Lour. & $\begin{array}{l}\text { M: Ka-ohn, Kaung- } \\
\text { oat-tee, Tha-phan; P: } \\
\text { Pha-owl }\end{array}$ & Food: ripe fruits edible & $\begin{array}{l}\text { TS0042, TS0110, TS0182 (M); } \\
\text { TS0461(P) }\end{array}$ \\
\hline Ficus racemosa L. & E, M, P: Tha-phan & $\begin{array}{l}\text { Food: ripe fruit edible, fresh fruits soaked in salty water, } \\
\text { pounded leaves as paste, tender leaves as salad, leaves cooked } \\
\text { with potato }\end{array}$ & TS00148 (M) \\
\hline $\begin{array}{l}\text { Ficus semicordata Buch.- } \\
\text { Ham. ex Sm. }\end{array}$ & $\begin{array}{l}\text { E, M, P: Ka-dut; P: } \\
\text { Tha-phan }\end{array}$ & $\begin{array}{l}\text { Food and Fuelwood: ripe fruit edible, fresh fruits fermented for } \\
\text { food, tender leaves for soup }\end{array}$ & $\begin{array}{l}\text { TS0071, TS00131, TS00172 (M); } \\
\text { TS0474 (P); TS0788 (E) }\end{array}$ \\
\hline Ficus virens Aiton & M, P: Nyaung-chin & Food: leaves and shoots for soup and salad & TS0140 (M) \\
\hline $\begin{array}{l}\text { Maclura fruticosa } \\
\text { (Roxb.) Corner }\end{array}$ & P: Sue-sein & Food: shoot as vegetable for soup and salad & TS0478 (P) \\
\hline \multicolumn{4}{|l|}{ Myricaceae } \\
\hline $\begin{array}{l}\text { Myrica esculenta Buch.- } \\
\text { Ham. ex D. Don }\end{array}$ & M: Kata-pho & Food: ripe fruits edible & TS0058, TS0109, TS0180 (M) \\
\hline \multicolumn{4}{|l|}{ Myrtaceae } \\
\hline Psidium guajava $\mathrm{L}$. & E: Mar-la-kar & Food: fruits eaten fresh & TS0760 (E) \\
\hline $\begin{array}{l}\text { Syzygium cumini (L.) } \\
\text { Skeels }\end{array}$ & E, M, P: Tha-pyay & Food and Construction: ripe fruits edible & TS0215 (M) \\
\hline $\begin{array}{l}\text { Syzygium oblatum } \\
\text { (Roxb.) Wall. ex } \\
\text { A.M.Cowan \& Cowan }\end{array}$ & E, M, P: Tha-pyay & Food and Construction: ripe fruits edible & TS0253 (M) \\
\hline $\begin{array}{l}\text { Syzygium pycnanthum } \\
\text { Merr. \& L.M.Perry }\end{array}$ & E, M, P: Tha-pyay & Food, Construction and Fuelwood: ripe fruits edible & TS0104 (M); TS0615 (P) \\
\hline \multicolumn{4}{|l|}{ Oleaceae } \\
\hline Anacolosa clarkii Pierre & M: Tay-pin & Food: fruits edible & TS0259 (M) \\
\hline \multicolumn{4}{|l|}{ Oxalidaceae } \\
\hline Oxalis latifolia Kunth & M: Mu-chin & Food: children eat all parts of plants & TS0024 (M) \\
\hline \multicolumn{4}{|l|}{ Phyllanthaceae } \\
\hline Antidesma acidum Retz. & E: Kim-ma-lin & Food: fruits and leaves as vegetable & TS0795 (E) \\
\hline $\begin{array}{l}\text { Bischofia javanica } \\
\text { Blume }\end{array}$ & M: Yae-pa-done & Food: shoots for salad, young leaves as vegetable & TS0041, TS0074 (M) \\
\hline Phyllanthus emblica $\mathrm{L}$. & $\begin{array}{l}\text { E: Zepyu; M: Se-sar; } \\
\text { P: Se-shar }\end{array}$ & $\begin{array}{l}\text { Food, Fuelwood and Medicine: fruits pounded as salad, boiled } \\
\text { fruits pounded and prepared salad, consumed as food for } \\
\text { hypertension, fruits eaten fresh for over bleeding, fruits roasted } \\
\text { and consumed as food for cough }\end{array}$ & $\begin{array}{l}\text { TS0033, TS0052, TS00213, TS0185, } \\
\text { TS00280 (M); TS0351, TS0670 (P); } \\
\text { TS0806 (E) }\end{array}$ \\
\hline
\end{tabular}

Plantaginaceae

Plantago major L. 
Table 2 List of identified wild edible plants used by villagers in this study (Continued)

\begin{tabular}{|c|c|c|c|}
\hline Taxon & $\begin{array}{l}\text { Village and } \\
\text { vernacular name }\end{array}$ & Use & Voucher number (Collection site ${ }^{a}$ ) \\
\hline & & $\begin{array}{l}\text { used as bandage for broken legs, young leaves prepared as } \\
\text { salad, consumed as food for toxemia and hypertension; paste of } \\
\text { entire plant used over abscess }\end{array}$ & \\
\hline \multicolumn{4}{|l|}{ Primulaceae } \\
\hline Embelia ribes Burm.f. & M, P: Kan-pa-lar & $\begin{array}{l}\text { Food: leaves as salad and soup, tender leaves and buds boiled } \\
\text { and eaten with fish sauce }\end{array}$ & TS0175 (M) \\
\hline \multicolumn{4}{|l|}{ Rhamnaceae } \\
\hline Ziziphus incurva Roxb. & P: Sue-kauk & Food: fruits edible & TS0688 (P) \\
\hline \multicolumn{4}{|l|}{ Rosaceae } \\
\hline $\begin{array}{l}\text { Docynia indica (Wall.) } \\
\text { Decne. }\end{array}$ & M, P: Pin-sein & $\begin{array}{l}\text { Food and Fuelwood: fruits eaten fresh, cooked as soup, } \\
\text { pounded with chili as side dish }\end{array}$ & $\begin{array}{l}\text { TS0032, TS0133, TS0168, TS0204 } \\
\text { (M); TS0399, TS0429, TS0513, TS } \\
\text { 0588, TS0638 (P) }\end{array}$ \\
\hline \multicolumn{4}{|l|}{ Rubiaceae } \\
\hline Coffea arabica L. & P: Coffee & Food: roasted seed for coffee & TS0519 (P) \\
\hline $\begin{array}{l}\text { Wendlandia budleioides } \\
\text { Wall. ex Wight \& Arn. }\end{array}$ & M, P: Thit-ne & Food and Construction: tender leaves for salad & TS0085 (M) \\
\hline $\begin{array}{l}\text { Wendlandia tinctoria } \\
\text { (Roxb.) DC. }\end{array}$ & M, P: Thit-ni & Food and Fuelwood: tender leaves as vegetable & TS0176 (M) \\
\hline \multicolumn{4}{|l|}{ Rutaceae } \\
\hline $\begin{array}{l}\text { Casimiroa cf. edulis La } \\
\text { Llave }\end{array}$ & M, P: Tha-gyar-tee & Food: fruits edible & TS0044 (M) \\
\hline $\begin{array}{l}\text { Clausena excavata } \\
\text { Burm.f. }\end{array}$ & M: Pyin-thaw-sein & Food: tender leaves as raw salad & TS0069 (M) \\
\hline $\begin{array}{l}\text { Murraya koenigii (L.) } \\
\text { Spreng. }\end{array}$ & P: Pyin-taw-thein & Food: tender leaves as raw salad & TS0543 (P) \\
\hline $\begin{array}{l}\text { Zanthoxylum armatum } \\
\text { DC. }\end{array}$ & M: Mike-cup & Food: tender leaves ingredient to beef curry & TS0030 (M) \\
\hline \multicolumn{4}{|l|}{ Salicaceae } \\
\hline $\begin{array}{l}\text { Casearia graveolens } \\
\text { Dalzell }\end{array}$ & E: Phan-khar & Food and Construction: fruits eaten fresh & TS0801(E) \\
\hline \multicolumn{4}{|l|}{ Sapindaceae } \\
\hline $\begin{array}{l}\text { Choerospondias axillaris } \\
\text { (Roxb.) B.L.Burtt \& } \\
\text { A.W.Hill }\end{array}$ & E: Del-cline & Food: fruits edible & TS0765 (E) \\
\hline $\begin{array}{l}\text { Dimocarpus fumatus } \\
\text { (Blume) Leenh. }\end{array}$ & E: Taw-kyat-mauk & Food: fipe fruits edible & TS0803 (E) \\
\hline $\begin{array}{l}\text { Spondias pinnata (L. f.) } \\
\text { Kurz }\end{array}$ & E: Gwe & Food: fruits eaten fresh & TS0812 (E) \\
\hline \multicolumn{4}{|l|}{ Schoepfiaceae } \\
\hline Schoepfia fragrans Wall. & P: Byauk-ole-kyi & Food: fruits edible & TS0610 (P) \\
\hline \multicolumn{4}{|l|}{ Smilacaceae } \\
\hline $\begin{array}{l}\text { Smilax gagnepainii } \\
\text { T.Koyama }\end{array}$ & $\begin{array}{l}\text { M: Sue-yit-sein; P: } \\
\text { Sue-yit }\end{array}$ & Food: shoots fried as vegetable, cooked soup, green salad & TS0617 (P) \\
\hline \multicolumn{4}{|l|}{ Solanaceae } \\
\hline Physalis angulata $\mathrm{L}$. & E: no name & Food: fruits eaten fresh & TS0756 (E) \\
\hline Physalis pubescens $\mathrm{L}$. & P: Taw-kha-yan-chin & Food: fruits eaten fresh & TS0477 (P) \\
\hline Solanum torvum Sw. & $\begin{array}{l}\text { E, M: Kha-yan-ka-zot; } \\
\text { P: Ka-zot }\end{array}$ & Food: fruits as vegetable & TS0186 (M); TS0737 (E) \\
\hline \multicolumn{4}{|l|}{ Theaceae } \\
\hline Camellia taliensis & M, P: Taw-la-phat & Food: tender leaves for salad & TS0112 (M); TS0339 (P) \\
\hline
\end{tabular}


Table 2 List of identified wild edible plants used by villagers in this study (Continued)

\begin{tabular}{|c|c|c|c|}
\hline Taxon & $\begin{array}{l}\text { Village }{ }^{a} \text { and } \\
\text { vernacular name }\end{array}$ & Use & Voucher number (Collection site ${ }^{a}$ ) \\
\hline \multicolumn{4}{|l|}{ (W.W.Sm.) Melch. } \\
\hline Schima wallichii Choisy & M: Thit-yar & Food and Constructiion: shoots as vegetable & TS0086 (M) \\
\hline \multicolumn{4}{|l|}{ Urticaceae } \\
\hline $\begin{array}{l}\text { Dendrocnide } \\
\text { basirotunda (C.Y.Wu) } \\
\text { Chew }\end{array}$ & E: Tha-phan & Food: ripe fruits edible & TS0780 (E) \\
\hline \multicolumn{4}{|l|}{ Vitaceae } \\
\hline $\begin{array}{l}\text { Leea indica (Burm. f.) } \\
\text { Merr. }\end{array}$ & $\begin{array}{l}\text { E: no name; M: Pait- } \\
\text { chin }\end{array}$ & Food: shoot as vegetable & TS0226 (M); TS0805 (E) \\
\hline \multicolumn{4}{|l|}{ Zingiberaceae } \\
\hline $\begin{array}{l}\text { Alpinia nigra (Gaertn.) } \\
\text { Burtt }\end{array}$ & M: Gon-min & Food: pith as vegetable & TS0278 (M) \\
\hline $\begin{array}{l}\text { Curcuma cf. amada } \\
\text { Roxb }\end{array}$ & M: Ba-thae-kaw & $\begin{array}{l}\text { Food and Medicine: dry powder of rhizome consumed orally for } \\
\text { flatulence, pounded fresh rhizome as spice in traditional curry, } \\
\text { rhizome sliced and dry and made powder, and used as spice in } \\
\text { curry }\end{array}$ & TS0068 (M); TS0727 (E) \\
\hline $\begin{array}{l}\text { Curcuma aromatica } \\
\text { Salisb. }\end{array}$ & M: Mar-lar-pu & $\begin{array}{l}\text { Food and Medicine: buds as vegetable, paste of rhizome used } \\
\text { externally over injury }\end{array}$ & TS0210/1 (M) \\
\hline
\end{tabular}

a: E, Eden; M, Myin Ka; P, Pin-sein-pin

Four species from three families were used as spices and condiments in traditional curry. The dry powder of the rhizome from Curcuma cf. amada was used in various traditional dishes. Freshly pounded rhizome of $C$. cf. amada was also used as a spice. Leaves of Cinnamomum tamala, Laurus cf. nobilis and Zanthoxylum armatum were used in traditional curry for flavor. $Z$. armatum is also used as a spice in Yunnan, the province of China nearest to Myanmar [36].

The different methods of preparation of vegetables for traditional dishes are as follows: (i) Hin, the most

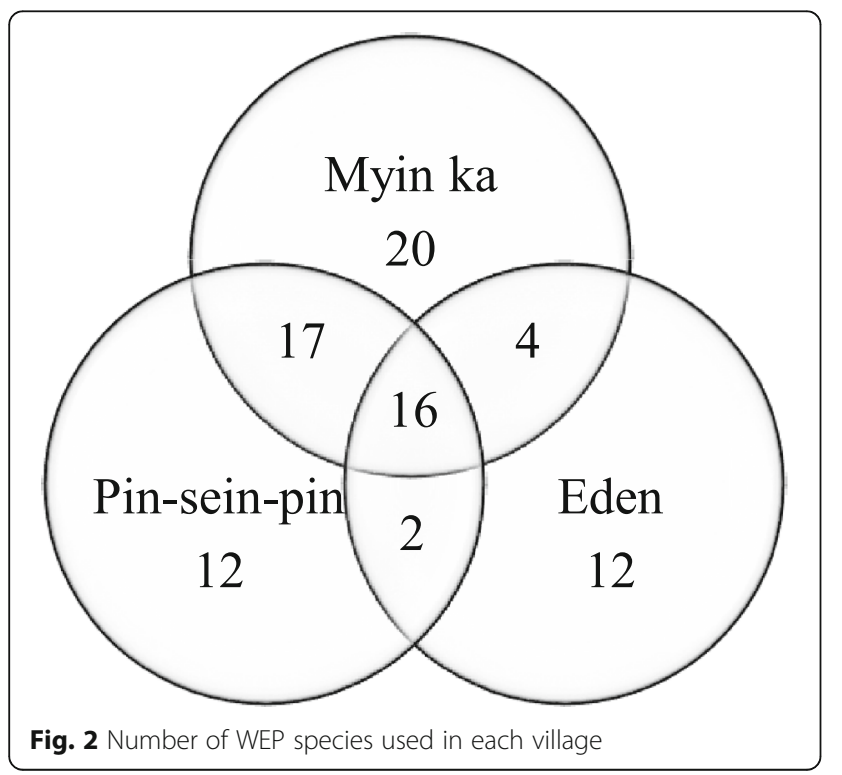

common traditional food, is prepared by boiling the vegetable with edible oil, fermented fish and salt into a semiliquid dish; (ii) Akyaw is prepared by frying the vegetable with salt, spices, chili, and fermented fish; (iii) Athote is prepared by mixing the raw or boiled vegetable with pounded fried groundnut, salt and chili; (iv) Atoe is a raw salad eaten with fermented fish or with fermented bean; and (v) Hincho is a soup prepared by boiling the vegetable with fermented fish and salt.

Most of the WEPs in this study were used directly as vegetable dishes without any pretreatments, although it is known that some plant species, such as those in Arisaema and Celastrus, are toxic. Only the tubers of Amorphophallus purpurascens and A. cf. muelleri were boiled, peeled and pounded before preparing traditional dishes. Most of the fruits, which were consumed as a snack food, were eaten fresh without any preparation. The fresh fruits of Ficus racemosa were preserved by soaking them in salty water. The petiole of Colocasia esculenta and the fruits of $F$. semicordata were pickled and consumed as a fermented food similar to pickles.

\section{Use of WEPs as medicine}

A total of 18 WEPs from 15 families were used as medicine (Table 3). Among these species, 11 were consumed as food for medicinal uses, and seven species were prepared as a main ingredient for traditional medicine. Dichrocephala integrifolia, Plantago major and Laggera alata were rarely used as food in the study area; however, these species were traditionally consumed for medicinal use. In the study area, the shoots and leaves of $D$. 
integrifolia were prepared as soup and consumed as a postpartum tonic for mothers. This species is also reported as a valuable medicinal plant for the treatment of Alzheimer's disease [37]. The young leaves of $P$. major were traditionally prepared as salad and consumed for hypertension and food poisoning. It is reported that the extract from $P$. major has antifungal activity [38]. The shoots of $L$. alata were fried with eggs and consumed to treat swelling in the body. Isochlorogenic acid A from L. alata is reported as a potential candidate antihepatitis B drug [39].

\section{Discussion}

\section{Species identification}

We applied the DNA barcoding technique as a guide for species identification. The chloroplast $r b c L$ sequences of 71 species ( $85.5 \%$ of 83 identified species) were successfully sequenced. The sequences of 53 species $(71.6 \%$ of sequenced species) yielded a single haplotype from a particular species from the BLAST analysis, whereas the remaining sequences yielded two or more haplotypes from closely related genera. As many of the collected specimens were sterile plants, the DNA barcoding technique facilitated the identification of the WEPs in this study.

\section{Notable uses of WEPs}

Lasia spinosa and Cheilocostus speciosus were important seasonal foods for the cash income of the local people. The villagers collected these two species from the wild seasonally and sold them in the local markets. Because customers prefer these two species as wild plants, the villagers do not cultivate them for markets (Fig. 3a). Tender leaves of Acacia concinna were used as vegetables, but the decoction of fruits of Acacia concinna is traditionally and popularly used as a shampoo in Myanmar. The stems and tubers of Amorphophallus purpurascens and Amorphophallus cf. muelleri were used as vegetables, and the sliced tubers were preserved after being sun dried. The tubers of these species were boiled, peeled and pounded to make konjac, referred to as 'Wa-u' in Myanmar (Fig. 3b). Boiled seeds of Archidendron jiringa are a traditional food in the area, and the seeds are also a source of income for the villagers. Although the boiled seeds are consumed widely in the tropics, they have also been found to have some toxicological effects on the heart, kidney, liver and pancreas [40].

Table 3 List of wild edible plants used as medicinal plants

\begin{tabular}{|c|c|c|}
\hline Taxon & Use & Ailment \\
\hline $\begin{array}{l}\text { Alternanthera } \\
\text { philoxeroides }\end{array}$ & Food as medicine & Inflamation \\
\hline Arisaema erubescens & Food as medicine & Digestive system disorders \\
\hline Celastrus paniculatus & Food as medicine & Nutritional Disorders \\
\hline Centella asiatica & $\begin{array}{l}\text { Ingredient for } \\
\text { medicine }\end{array}$ & Respiratory system disorders; Sensory system disorders \\
\hline Cheilocostus speciosus & $\begin{array}{l}\text { Ingredient for } \\
\text { medicine }\end{array}$ & Digestive system disorders \\
\hline Cinnamomum tamala & $\begin{array}{l}\text { Ingredient for } \\
\text { medicine }\end{array}$ & Birth related disorders \\
\hline Colocasia esculenta & $\begin{array}{l}\text { Ingredient for } \\
\text { medicine }\end{array}$ & Poison (Insect poison) \\
\hline Curcuma aromatica & $\begin{array}{l}\text { Ingredient for } \\
\text { medicine }\end{array}$ & Injuries \\
\hline Curcuma cf. amada & $\begin{array}{l}\text { Ingredient for } \\
\text { medicine }\end{array}$ & Digestive system disorders \\
\hline Dichrocephala integrifolia & Food as medicine & Birth related disorders \\
\hline Laggera alata & $\begin{array}{l}\text { Ingredient for } \\
\text { medicine }\end{array}$ & Inflamation; Pains; Injuries \\
\hline Macropanax dispermus & Food as medicine & Digestive system disorders \\
\hline Momordica subangulata & Food as medicine & Nutritional disorders \\
\hline Oroxylum indicum & Food as medicine & Sensory system disorders \\
\hline Phyllanthus emblica & Food as medicine & Circulatory system disorders; Respiratory system disorders \\
\hline Plantago major & Food as medicine & $\begin{array}{l}\text { Poison (Food poison); Skin/Subcutaneous/Cellular tissue disorders; Injuries; Circulatory system } \\
\text { disorders }\end{array}$ \\
\hline Rotheca serrata & Food as medicine & Digestive system disorders; Mental disorders \\
\hline Telosma cordata & Food as medicine & Mental disorders \\
\hline
\end{tabular}




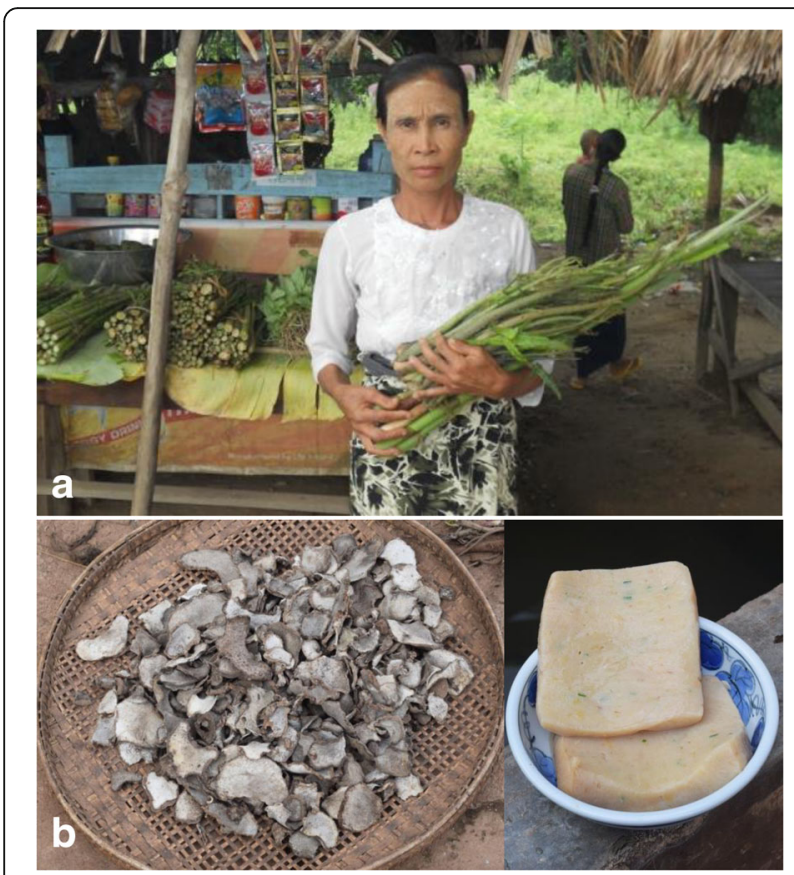

Fig. 3 Use of WEPs in the study area. a wild Lasia spinosa and Cheilocostus speciosus at a local roadside market. Informed consent was obtained for the use of the photograph. $\mathbf{b}$ sun-dried slices of Amorphophallus purpurascens for preservation (left) and 'Wa-u' (right)

The seeds of Elaeocarpus floribundus were used to extract vegetable oil in Myin Ka village. The vegetable oil was used locally, but it was not produced at a commercial scale. To the best of our knowledge, there are no reports on the use of seed oil of E. floribundus, although its fruits are eaten raw as a wild edible fruit in South Asia [41]. It is interesting that the extracts of leaves of $E$. floribundus showed significant activities against CEM-SS cancer cells [42].

It is reported that Rotheca serrata is mainly used as a medicinal plant in other areas [43]. However, R. serrata was mainly consumed as a wild vegetable in the study area and was used for digestive system disorders. The young leaves of Celastrus paniculatus were prepared in a traditional soup and consumed as a dietetic food. The food is not used for a particular ailment. However, the villagers believe that the soup is good for health. The use of this species was recorded in a traditional song expressing that the plant is highly recommended for traditional soup. The fruits of $C$. paniculatus are consumed orally as a vermifuge by the locals living around Popa Mountain Park, Myanmar [44]. It was reported that the extract from $C$. paniculatus can inhibit the growth of breast cancer cells [45].

\section{WEPs used in each village}

The differences in the use of WEPs between the communities of three villages have been observed. The differences among ethnic groups could be the main reason for using different local names for the same species. The three villages are situated in different townships, and there are no social relationships among the villages. This is also another reason for using different local names.

The village of Myin Ka used a larger number of WEPs than the other two villages. Myin Ka village has a longer history than the other two villages and could have inherited much more traditional knowledge of WEPs. In spite of the same major ethnic group (Danu people) and similar vegetation being found in the villages of Myin Ka and Pin-sein-pin, 33 out of 71 WEP species were used in both of the villages, but the remaining 38 species were not common between the two villages. These two communities have different historical backgrounds, and there were no social relationships between the two villages. These differences may be a cause of different uses of the plant resources.

The village of Eden has fewer shared species with the other two villages. Most of the species documented in this study from the families Ebenaceae, Elaeagnaceae, Elaeocarpaceae, Fagaceae, Lamiaceae, Lauraceae, Myricaceae, Rosaceae, Rubiaceae and Rutaceae were used in the villages of Myin Ka and Pin-sein-pin, but not in the village of Eden. However, most species from the families Burseraceae, Lythraceae and Sapindaceae were used only in Eden village. The elevation of Eden is approximately $350 \mathrm{~m}$ above sea level, while that of Myin Ka and Pin-sein-pin is more than $1000 \mathrm{~m}$. The forests around the village of Eden are mostly deciduous forests, while those around the villages of Myin Ka and Pin-sein-pin are evergreen forests. These differences could be the causes of variation in plants used by the local communities. Moreover, the village of Eden is newly established by Kayan people who migrated from Kayah State, which is different from the villages of Myin Ka and Pin-sin-pin, which were established by Danu people in Shan State. The migrant community may be less familiar with the plant resources in the new environment. In addition, the villagers of Eden practice shifting cultivation for banana plantations. Shifting cultivation could have detrimental effects on the environment, and the availability of WEPs could be diminished.

The average number of species recognized by a single key informant was different among the three villages (Table 4). The key informants from Myin Ka village reported a greater number of species (an average of 18.0 species per key informant) than did those from the villages of Eden and Pin-sein-pin (an average of 10.2 and 12.1 species, respectively). The amount of knowledge on WEPs was also different between the age classes of key informants in the study area. Usually, the older generations transfer the farming activities and knowledge of WEPs to the younger generations in the study area. However, the 
Table 4 The numbers of wild edible plants recognized by each key informant

\begin{tabular}{|c|c|c|c|c|c|c|}
\hline \multirow[t]{2}{*}{ Age } & \multicolumn{2}{|l|}{ Eden } & \multicolumn{2}{|l|}{ Myin Ka } & \multicolumn{2}{|l|}{ Pin-sein-pin } \\
\hline & Informant & No. of WEPs recognized & Informant & No. of WEPs recognized & Informant & No. of WEPs recognized \\
\hline \multirow[t]{3}{*}{$30-39$} & $A$ & 12 & $\mathrm{~F}^{\mathrm{a}}$ & 21 & M & 22 \\
\hline & & & G & 28 & $N$ & 7 \\
\hline & & & $\mathrm{H}$ & 30 & & \\
\hline \multirow[t]{3}{*}{$40-49$} & B & 11 & । & 12 & $\mathrm{O}$ & 8 \\
\hline & C & 17 & J & 13 & $P$ & 9 \\
\hline & $\mathrm{D}$ & 5 & & & Q & 10 \\
\hline \multirow[t]{2}{*}{$50-59$} & $E$ & 6 & K & 9 & $\mathrm{R}$ & 18 \\
\hline & & & L & 13 & S & 11 \\
\hline Mean \pm SD & & $10.2 \pm 4.9$ & & $18.0 \pm 8.4$ & & $12.1 \pm 5.6$ \\
\hline
\end{tabular}

${ }^{\mathrm{a}}$ : female

age class of 30-39 years reported more WEPs than did the older groups. The interviews revealed that the age class of 30-39 years was much more familiar with the environments where they could collect WEPs. The sharing of work between family members was revealed, and the age class of 30-39 years took more responsibilities in the collection of WEPs. The older generations might face the inability to recall the knowledge of gathering WEPs. The abundance of knowledge of WEPs in younger age classes and a decrease in the knowledge exhibited by the older groups have been reported previously [46, 47].

The number of species reported as WEPs by more than two key informants in the villages of Eden, Myin $\mathrm{Ka}$ and Pin-sein-pin was 8 (33.3\% of all species reported by all key informants in Eden), 29 (56.9\%) and 15 (40.5\%) species, respectively. This result indicates more share knowledge of WEPs within the community of Myin Ka. Myin Ka village has 120 ha of common land for watershed conservation, and all villagers of Myin Ka village have access to the common land for collection of WEPs. In contrast, most of the land area around Pin-sein-pin village is privately owned. In the case of Eden village, shifting cultivation is practiced in the reserve forests, where the villagers do not have the legal right of land ownership. Thus, land use and the land ownership system could have effects on the sharing of knowledge of WEPs among the villagers.

\section{Conclusion}

The rich diversity and traditional knowledge of WEPs have been documented in this study. This study indicates that WEPs play an important role in the livelihood of local communities. Historical background, land use system and surrounding vegetation could have effects on the variation in the traditional uses of WEPs. The indigenous societies, especially that in the village of Myin $\mathrm{Ka}$, maintained a considerable amount of traditional knowledge of WEPs. In addition to their food value, other uses of WEPs, such as their medicinal uses, make them more important in the livelihood of local people. WEPs are important not only for increasing the diversity of local food consumption but also for generating income in the local communities. Increasing awareness of the importance of WEPs will encourage the conservation of traditional knowledge of indigenous populations. Further investigations on the nutritional value and pharmacological activities of WEPs will add more value to the traditional knowledge.

\section{Abbreviations \\ MBK: Makino botanical garden; WEP: Wild edible plant}

\section{Acknowledgments}

Special thanks are due to the villagers of Eden, Myin Ka and Pin-sein-pin for their crucial assistance. We are grateful to Dr. Nyi Nyi Kyaw, Director General of Forest Department, Myanmar, for giving us permission to carry out field research in Myanmar. We are also thankful to the staff members of the Forest Department of Kalaw and Pindaya Township for their help in the field research. This research is funded by a Ph.D. scholarship program of the Kochi Prefectural Makino Botanical Garden, Japan.

\section{Funding}

See acknowledgments.

\section{Authors' contributions}

TS carried out the field research and laboratory experiments and wrote the first draft of the manuscript; AZM provided assistance and suggestions for field research; FK provided suggestions and help in the field research and botanical identification; and HU conceived and designed the research and provided close supervision in all steps. All the authors read and approved the final manuscript.

Ethics approval and consent to participate

Not applicable.

Consent for publication

Not applicable.

Competing interests

The authors declare that they have no competing interests.

\section{Publisher's Note}

Springer Nature remains neutral with regard to jurisdictional claims in published maps and institutional affiliations. 


\section{Author details}

${ }^{1}$ College of Bioresource Sciences, Nihon University, 1866 Kameino, Fujisawa, Kanagawa 252-0880, Japan. ${ }^{2}$ Forest Department, Ministry of Natural Resource and Environmental Conservation, Forest Research Institute, Yezin, Naypyitaw, Myanmar. ${ }^{3}$ Kochi Prefectural Makino Botanical Garden, 4200-6, Godaisan, Kochi 781-8125, Japan. ${ }^{4}$ Ministry of Natural Resource and Environmental Conservation, Forest Research Institute, Yezin, Naypyitaw, Myanmar.

\section{Received: 1 September 2017 Accepted: 5 July 2018}

\section{Published online: 17 July 2018}

\section{References}

1. Leonti M, Nebel S, Rivera D, Heinrich M. Wild gathered food plants in the European Mediterranean: a comparative analysis. Econ Bot. 2006;60:130-42.

2. Reyes-García V, Menendez-Baceta G, Aceituno-Mata L, Acosta-Naranjo R, Calvet-Mir L, Domínguez P, Garnatje T, Gómez-Baggethun E, MolinaBustamante M, Molina M, Rodríguez-Franco R, Serrasolses G, Vallès J, Pardode-Santayana M. From famine foods to delicatessen: interpreting trends in the use of wild edible plants through cultural ecosystem services. Ecol Econ. 2015:120:303-11.

3. Penafiel D, Lachat C, Espinel R, Van Damme P, Kolsteren P. A systematic review on the contributions of edible plant and animal biodiversity to human diets. EcoHealth. 2011;8:381-99.

4. Powell B, Thilsted SH, Ickowitz A, Termote C, Sunderland T, Herforth A. Improving diets with wild and cultivated biodiversity from across the landscape. Food Sci. 2015;7:535-54.

5. Grivetti LE, Ogle BM. Value of traditional foods in meeting macroand micronutrient needs: the wild plant connection. Nutr Res Rev. 2000;13:31-46.

6. Turner NJ, Luczaj LJ, Migliorini P, Pieroni A, Dreon AL, Sacchetti LE, Paoletti MG. Edible and tented wild plants, traditional ecological knowledge and agroecology. Crit Rev Plant Sci. 2011;30:198-225.

7. Das G, Patra JK, Singdevsachan SK, Gouda S, Shin HS. Diversity of traditional and fermented foods of the seven sister states of India and their nutritional and nutraceutical potential: a review. Front Life Sci. 2016;9:292-312.

8. Ju Y, Zhuo J, Liu B, Long C. Eating from the wild: diversity of wild edible plants used by Tibetans in Shangri-la region, Yunnan, China. J Ethnobiol Ethnomed. 2013;9:28.

9. Abbet C, Mayor R, Roguet D, Spichiger R, Hamburger M, Potterat O. Ethnobotanical survey on wild alpine food plants in lower and Central Valais (Switzerland). J Ethnopharmacol. 2014;151:624-34.

10. Etkin NL, Ross PJ. Food as medicine and medicine as food. An adaptive framework for the interpretation of plant utilization among the Hausa of northern Nigeria. Soc Sci Med. 1982;16(17):1559-73.

11. Odhav B, Beekrum S, Akula U, Baijnath H. Preliminary assessment of nutritional value of traditional leafy vegetables in KwaZulu-Natal, South Africa. J Food Compos Anal. 2007;20(5):430-5.

12. Gu W, Ynag J, Yang FM, Sun QY, Wang YH, Long CL. A preliminary study of traditional, wild medicinal, edible plants in Xishuangbanna, Yunnan, China. Plant Divers Resour. 2014;36(1):99-108.

13. Turner NJ, Turner KL. Where our women used to get the food: cumulative effects and loss of ethnobotanical knowledge and practice; case study from coastal British Columbia. Botany. 2008;86:103-15.

14. Krupnick GA, Kress WJ. Hotspots and ecoregions: a test of conservation priorities using taxonomic data. Biodivers Conserv. 2003;12:2237-53.

15. Myers N, Mittermeier RA, Mittermeier CG, de Fonseca GAB, Kent J. Biodiversity hotspots for conservation priorities. Nature. 2000;403:853-8.

16. Hidayati S, Franco FM, Bussmann RW. Ready for phase 5 - current status of ethnobiology in Southeast Asia. J Ethnobiol Ethnomed. 2015;11:17.

17. Rao AN, Natesh S. Greater and urgent need for further research on ethnobotany and phytochemistry in south east Asian countries. J Trop Med Plants. 2008;9:153-8.

18. Ministry of Immigration and Population, Myanmar. The 2014 Population and Housing Census of Myanmar. Frequently Asked Questions and Answers; 2014.

19. Food and Agriculture Organization of the United Nations. Global Forest resources assessment 2010. FAO Forestry Paper 163; 2010.

20. Millennium Ecosystem Assessment. Ecosystems and human well-being: biodiversity synthesis. Washington, DC: World Resources Institute; 2005.

21. Peters CM, Henderson A, Maung UM, Lwin US, Ohn UTM, Lwin UK, Shaung UT. The rattan trade of northern Myanmar: species, supplies, and sustainability. Econ Bot. 2007;61:3-13.
22. Ono K, Suzuki K. Assessment of subsistence plant resource of the mangrove forest in the Ayeyarwady Delta, Myanmar. Global Environ Res. 2013;17:223-32.

23. Aung $H T$, Sein MM, Aye MM, Thu ZM. A review of traditional medicinal plants from Kachin State, northern Myanmar. Nat Prod Commun. 2016;1 1(3):353-64.

24. Khin Thantsin, San San Aye and Khin Thandar Win. Pharmaceutical ethnobotany survey of anticancer plant resources from the Hlawgar Wildlife Park, Yangon Region, Myanmar. 2016. http://uyr.uy.edu.mm/handle/ $123456789 / 180$

25. Hadden RL. The geology of Burma (Myanmar): an annotated bibliography of Burma's geology. Geogr Earth Sci. 2008. http://www.dtic.mil/dtic/tr/ fulltext/u2/a487552.pdf.

26. Hebert PDN, Cywinska A, Ball SL, de Waard JR. Biological identifications through DNA barcodes. Proc R Soc Lond B. 2003;270:313-21.

27. von Cräutlein $M$, Korpelainen $H$, Pietiläinen $M$, Rikkinen J. DNA barcoding: a tool for improved taxon identification and detection of species diversity. Biodivers Conserv. 2011;20:372-89.

28. Kang J, Kang Y, Ji X, Guo Q, Jacques G, Pietras M, Łuczaj N, Li D, Łuczaj Ł. Wild food plants and fungi used in the mycophilous Tibetan community of Zhagana (Tewo County, Gansu, China). J Ethobiol Ethnomed. 2016;12:21.

29. Kasper-Pakosz R, Pietras M, Łuczaj Ł. Wild and native plants and mushrooms sold in the open-air markets of south- eastern Poland. J Ethobiol. Ethnomed. 2016;12:45.

30. Tobe $H$, Shinohara W, Utami N, Wiriandinata H, Girmansyah D, Oginuma K Azuma H, Tokuoka T, Kawaguchi E, Kono M, Ito M. Plant diversity on Lombok Island, Indonesia: an approach at identification using DNA barcodes. Acta Phytotaxon Geobot. 2010;61(2):93-108.

31. International Society of Ethnobiology. ISE code of Ethics. 2008;

32. Phillips O, Gentry AH. The useful plants of Tambopata, Peru: I. Statistical hypotheses tests with a new quantitative technique. Econ Bot. 1993;47(1):15-32.

33. CBOL Plant Working Group. A DNA barcode for land plants. Proc Natl Acad Sci U S A. 2009;106:12794-7.

34. Cook FEM. Economic botany, data collection standard. Kew: Royal Botanic Gardens; 1995.

35. Holm L, Doll J, Holm E, Pancho JV, Herberger JP. World weeds: natural histories and distribution. New York: John Wilsey \& sons; 1997.

36. Shukri R, Mohamed S, Mustapha NM, Hamid AA. Evaluating the toxic and beneficial effects of jering beans (Archidendron jiringa) in normal and diabetic rats. J Sci Food Agric. 2011;91:2697-706.

37. Kouémou NE, Taime GS, Moto FCO, Pale S, Ngoupaye GT, Njapdounke JSK, et al. Nootropic and neuroprotective effects of Dichrocephala integrifolia on scopolamine mouse model of Alzheimer's disease. Front Pharmacol. 2017;8:1-10.

38. Shirley KP, Windsor LJ, Eckert GJ, Richard LG. In vitro effects of Plantago major extract, aucubin and baicalein on Candida albicans bioflim formation, metabolic activity and cell surface hydrophobicity. J Prosthodont. 2017; 26(6):508-15.

39. Hao BJ, Wu YH, Wang JG, Hu SQ, Keil DJ, Hu HJ, Lou JD, Zhao Y. Hepatoprotective and antiviral properties of isochlorogenic acid a from Laggera alata against hepatitis B virus infection. J Ethnopharmacol. 2012;144:190-4.

40. Geng Y, Zhang Y, Ranjitkar S, Huai H, Wang Y. Traditional knowledge and its transmission of wild edibles used by the Naxi in Baidi Village, Northwest Yunnan province. J Ethnobiol Ethnomed. 2016;12:10.

41. Brahma S, Narzary H, Basumatary S. Wild edible fruits of Kokrajhar district of Assam, North-East India, Asian J. Plant Sci Res. 2013;3(6):95-100.

42. Utami R, Khalid N, Sukari MA, Rahmani M, Abdul AB, Dachriyanus. Phenolic contents, antioxidant and cytotoxic activities of Elaeocarpus floribundus Blume. Pak J Pharm Sci. 2013;26:245-50.

43. Patel JJ, Acharya SR, Acharya NS. Clerodendrum serratum (L.) moon. - a review on traditional uses, phytochemistry and pharmacological activities. J Ethnopharmacol. 2014;154:268-85.

44. Htwe KM, Tanaka N, Gale S, Murata J. The ethnobotanical Flora of Mt Popa, Central Myanmar. MAKINOA NS. 2007;6:63-93.

45. Weng JR, Yen MH, Lin WY. Cytotoxic constituents from Celastrus paniculatus induce apoptosis and autophagy in breast cancer cells. Phytochemistry. 2013:94:211-9.

46. Ahmad K, Pieroni A. Folk knowledge of wild food plants among the tribal communities of Thakhte-Sulaiman Hills, north-West Pakistan. J Ethnobiol Ethnomed. 2016;12:17.

47. Kang Y, Łuczaj $Ł$, Kang J, Zhang S. Wild food plants and wild edible fungi in two valleys of the Qinling Mountains (Shaanxi, Central China). J Ethnobiol Ethnomed. 2013;9:26. 